\title{
Analysis of Resistive Forces Acting on the Bucket of LHD in the Scooping Task
}

\author{
Hiroshi TAKAHASHI ${ }^{1}$, Yoshiaki TSUKAMOTO ${ }^{2}$ and Eiji NAKANO ${ }^{3}$ \\ 1. Dept. of Geoscience and Technology, Graduate School of Engineering, Tohoku University, \\ Sendai 980-8579, JAPAN \\ e-mail: htaka@ecml.earth.tohoku.ac.jp \\ 2. Furukawa Co.LTD, JAPAN \\ 3. Graduate School of Information Science, Tohoku University, JAPAN
}

\begin{abstract}
In order to automate the scooping task, it is desirable that the resistive force acting on the bucket be obtained before the scooping is performed. The objective of this study is to derive theoretically the resistive forces acting on the bucket during scooping of piled rock fragments. First, the changes in the shape of the rock pile due to the penetration of it by the bucket are modeled. Based on this model, the resistive forces acting on the bucket are considered theoretically. Finally, it was confirmed that the calculated results obtained using the model agree well with the experimental results.
\end{abstract}

\section{Introduction}

Load-Haul-Dump(LHD) is a machine which has been widely used in underground mines. Recently, the automation of $\mathrm{LHD}$ has been receiving considerable attention in order to release the operators from the severe working environment. LHD has three functions, loading, carrying and dumping of crushed rocks. Carrying of the crushed rocks has already been conducted by using the $\mathrm{CCD}$ camera vision system[1], ultrasonic range finders[2] or electromagnetic cables[3]. That is, navigation of LHD has been already successful. On the other hand, loading/scooping is much more difficult to automate because the interaction between the piled rock and the bucket is very complicated. However, the automatic scooping is necessary to establish the full automation of LHD tasks, and in order to establish the automatic scooping, it is desirable that the resistive force acting on the bucket be obtained before the scooping is performed.

Therefore, the purpose of this study is to investigate the resistive forces acting on the bucket of an LHD, theoretically and experimentally. Usually, the scooping task consists of two steps, that is, 1)penetration of the bucket into the rock pile and 2)lifting of the bucket to scoop the rocks. In this study, only the first step is considered.

\section{Visualization of the Changing Process of Piled} Rock Fragments

In order to analyze the resistive forces acting on the bucket, it is very important to know how the rock pile is changing due to the penetration of the bucket into the rock pile. Therefore, the first, visualization experiment was carried out to investigate the changing process in the shape of the rock pile.

Figure 1 shows the schematic diagram of the experimental apparatus. The rock pile was made at the comer of the acrylic box by using $5.7 \mathrm{~mm}$ diameter-crushed rocks. Some of the particles were painted with white coloring for easy visual observation. One side of the bucket was taken off and the bucket was pushed into the rock pile. The changing process in the shape of the rock pile was recorded with the video camera. Then the trajectories of the white particles were obtained by X-Y coordinator.

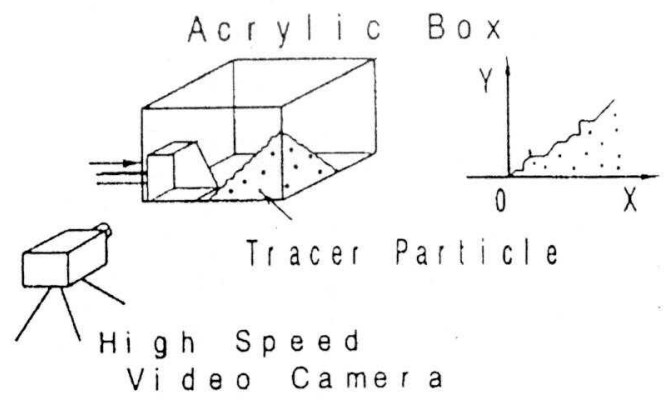

Fig.1 Schematic of visualization experimental apparatus

Figure 2 shows the trajectories of the white particles. It can be seen from this figure that particle trajectories can be classified into three parts, that is, 1)the part which moves horizontally with the bucket, 2)the part which moves upward and 3)the part which does not move.

The changing process in the shape of the rock pile 
was modeled based on the results shown in Figure 2 and the following assumptions:

1) The model is two dimensional. When the calculated results are compared with the experimental ones, the bucket width is taken into account.

2) The shape of the failure plane is flat.

3) The size of particles in the rock pile is approximately the same, and cohesion among particles is negligible.

4)The thickness of the bucket is negligibly small compared with the size of the rock pile.

5) The compression of the rock pile due to the penetration of the bucket is neglected.

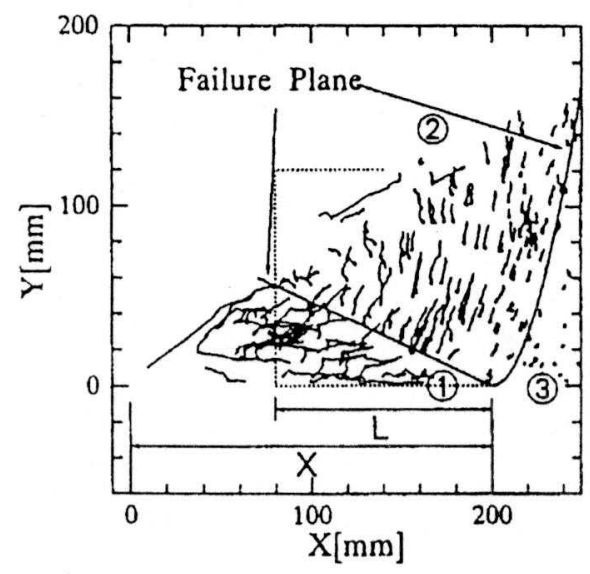

Fig.2 Trajectories of tracer particles
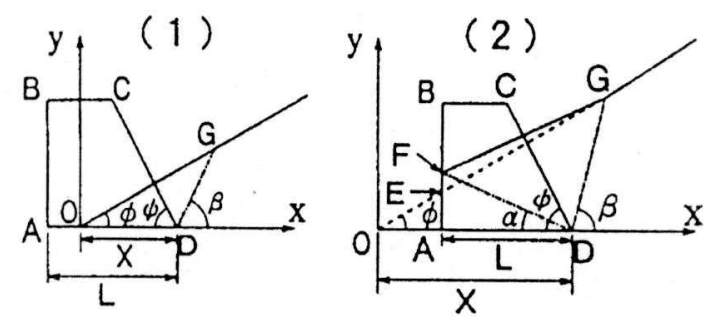

Fig. 3 Modeling of the changes in the shape of rock Pile

Figure 3 shows the model of the changing process in the shape of the rock pile. Diagrams (1) and (2) show the models for which the penetration distance of the bucket is less and larger than the bucket bottom length, respectively. $A D F$ and DFG correspond to 1)the part which moves horizontally with the bucket and 2)the part which moves upward in Figure 2, respectively.

With taking into account the previous study concerned with the resistive forces acting on the bucket of a back hoe, the resistive force acting on the bucket of LHD, F is assumed as Eq. (1) in this study.

$$
F=F_{1}+F_{2}+F_{3}
$$

$F_{1}$ : the resistive force due to the passive soil pressure $\mathrm{F}_{2}$ : friction force between the bucket and rock pilc $\mathrm{F}_{3}$ : cutting force

\section{a)Modeling of $F_{1}$}

In order to formulate $F_{1}$, Coulomb's soil theory is applied. In Coulomb's theory, the force due to the passive soil pressure is obtained by solving the force balance equations as shown in Figure 4. In Figure 4, the angle of the failure plane, $\beta$ is unknown. In the calculation, the minimum $F_{p}$ is sought by changing the angle of the failure plane, $\beta$. The minimum $F_{P}$ is the force due to the passive soil pressure.

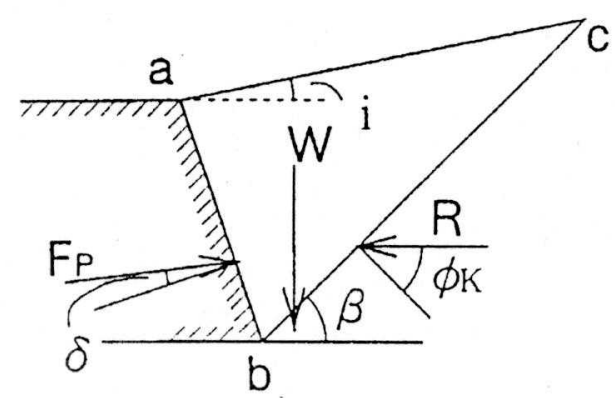

Fig. 4 Forces due to the passive soil pressures

Based on the results shown in Figure 2, the changes in shape of the rock pile were modeled as shown in Figure 5. From this model, $F_{1}$ is expressed as follows in the case of (1) and (2), respectively.
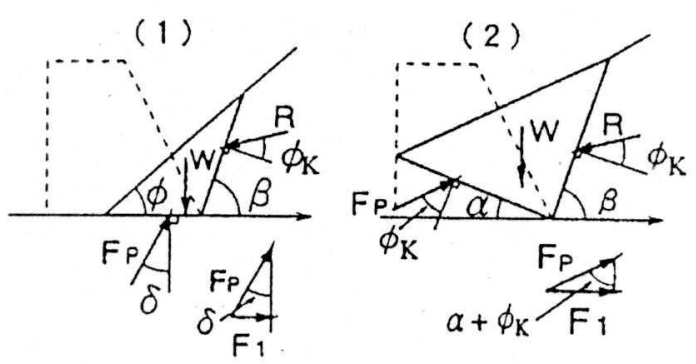

Fig.5 Forces acting on the bucket

In the case of (1):

$$
\begin{aligned}
& F_{1}=F_{P} \sin \delta \\
& F_{P}=W /\left\{\cos \delta+\sin \delta \frac{\cos \left(\beta+\phi_{k}\right)}{\sin \left(\beta+\phi_{k}\right)}\right\} \\
& W=S_{O D G} B \cdot \rho(1-\varepsilon)
\end{aligned}
$$

In the case of (2)

$$
\begin{aligned}
& F_{1}=F_{P} \sin \left(\alpha+\phi_{K}\right) \\
& F_{P}=W /\left\{\cos \left(\alpha+\phi_{k}\right)+\sin \left(\alpha+\phi_{k}\right) \frac{\cos \left(\beta+\phi_{k}\right)}{\sin \left(\beta+\phi_{k}\right)}\right\}
\end{aligned}
$$




$$
W=S_{D F G} B \cdot \rho(1-\varepsilon)
$$

\section{b)Modeling of $F_{2}$}

The resistive force due to the friction between the bucket and the rock pile is determined by the static pressure from the rock pile, friction coefficient between the bucket and the rock pile, rock density and porosity in the rock pile. By using the model shown in Figure 6, $F_{2}$ is expressed as follows in the case of (1) and (2), respectively.

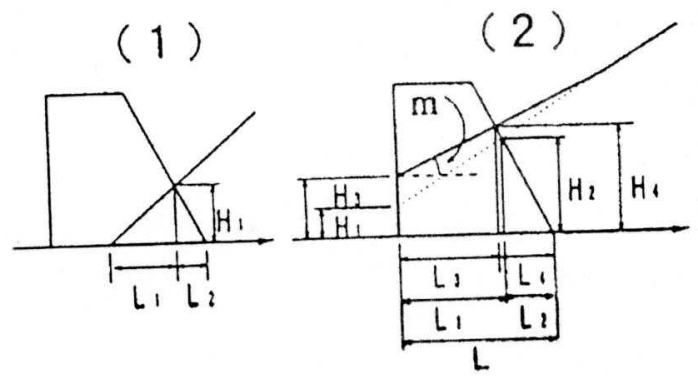

Fig.6 Definition of each length

In the case of (1)

$$
\begin{aligned}
& F_{2}=\left(F_{11}+F_{12}\right) \times 2 \\
& F_{11}= \frac{1}{2} \mu \rho(1-\varepsilon)\left(\frac{1}{3} \tan ^{2} \phi \cdot L_{1}{ }^{3}\right) \times 2 \\
& F_{12}=\frac{1}{2} \mu \rho(1-\varepsilon)\left\{-\frac{1}{3}(2 \tan \phi+\tan \psi) \tan \psi \cdot L_{2}{ }^{3}\right. \\
&\left.+H_{1} \tan \phi \cdot L_{2}{ }^{2}+H_{1}{ }^{2} L_{2}\right\} \times 2
\end{aligned}
$$

In the case of (2)

$$
\begin{aligned}
F_{2}= & \left(F_{21}+F_{22}+F_{23}+F_{24}-F_{25}\right) \times 2 \\
F_{21}= & \frac{1}{2} \mu \rho(1-\varepsilon)\left\{\frac{1}{3} \tan ^{2} \phi \cdot L_{1}{ }^{3}+H_{1} \tan \phi \cdot L_{1}{ }^{2}+H_{1}{ }^{2} L_{1}\right\} \\
F_{22}= & \frac{1}{2} \mu \rho(1-\varepsilon)\left\{-\frac{1}{3}(2 \tan \phi+\tan \psi) \tan \psi \cdot L_{2}{ }^{3}\right. \\
& \left.+H_{2} \tan \phi \cdot L_{2}{ }^{2}+H_{2}{ }^{2} L_{2}\right\}
\end{aligned}
$$

Here, $\phi$ is the slope of the rock pile, $\phi$ is the bucket angle, $\mu$ is the friction coefficient between the

c)Modeling of $F_{3}$

(1)

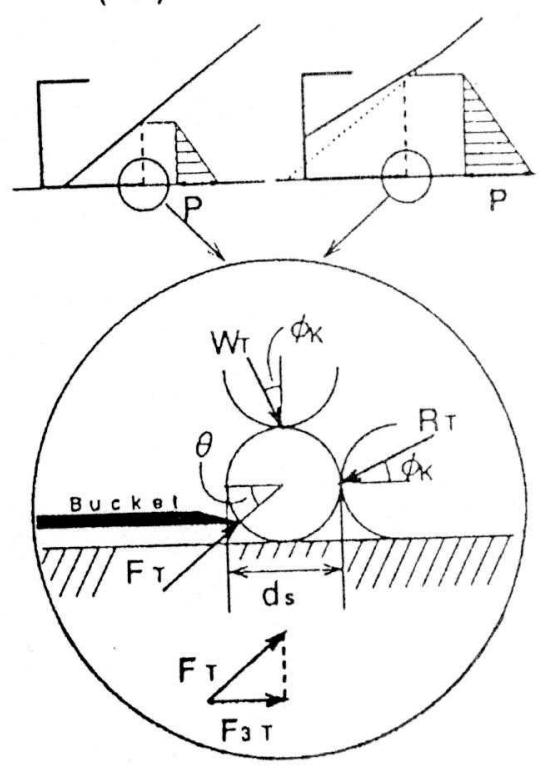

Fig.7 Particle behavior at the bucket tip
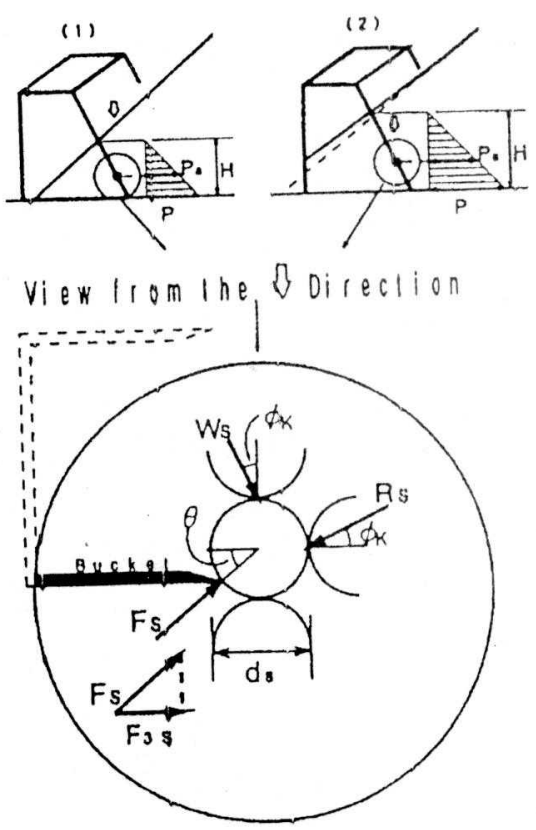

Fig.8 Particle behavior at the bucket side

Figure 7 shows the schematic diagram of the particle behavior at the bucket bottorn tip. As shown in this figure, it is assumed that the particle which is pushed by the bucket bottom tip moves upward with rotating. The forces acting on the particle are the pushing force by the buckct bottom, $\mathrm{F}_{\mathrm{T}}$, reactive force, 
$\mathrm{R}_{\mathrm{T}}$ and particle weight $\mathrm{W}_{\mathrm{T}}$. These forces are determined by solving the force balance equations in the horizontal and vertical directions. The cutting force is acting not only on the bucket bottom but also on the bucket side. The particle behavior at the bucket side is modeled as shown in Figure 8 . The forces acting on this particle are the pushing force by the bucket side, $F_{S}$, reactive force, $R_{S}$ and particle weight $\mathrm{W}_{\mathrm{S}} \mathrm{W}_{\mathrm{S}}$ is obtained by integrating the product of the pressure $\mathrm{P}_{\mathrm{a}}$ and particle diameter ds by height $\mathrm{H}$. By considering the above model, the cutting force, $\mathrm{F}_{3}$ is given as follows.

$$
F_{3}=F_{3 T}+2 \times F_{3 S}
$$

(Side of the bucket)

$$
\begin{aligned}
& F_{3 T}=F_{T} \cdot \cos \theta \\
& F_{T}=W_{T} \frac{\left(\cos \phi_{K}+\frac{\sin ^{2} \phi_{K}}{\cos \theta}\right)}{\sin \theta-\sin \phi_{K}} \\
& W_{T}=\frac{P \cdot B \cdot d_{s}}{\cos \phi_{K}}
\end{aligned}
$$

(Bottom of the bucket) )

$$
\begin{aligned}
& F_{3 S}=F_{S} \cdot \cos \theta \\
& F_{S}=W_{S} \frac{\left(\cos \phi_{K}+\frac{\sin ^{2} \phi_{K}}{\cos \theta}\right)}{\sin \theta-\sin \phi_{K}} \\
& W_{S}=\frac{P \cdot H^{2}}{2 \cdot \cos \phi_{K}}
\end{aligned}
$$

Here, $\phi_{\mathrm{k}}$ is the friction angle among particles; $\theta$ is the angle between the line connecting the bucket tip and particle center and the horizontal line. ds is the particle diameter, $\mathrm{B}$ is the bucket width, and $\mathrm{H}$ is the height as shown in Figure 8. $\theta$ is an unknown parameter, and this value will be determined experimentally.

\section{Experimental Apparatus}

Figure 9 shows the schematic diagram of the experimental apparatus. As the resistive force acting on the bucket is a function of the penetration distance of the bucket into the rock pile, it is necessary to measure the resistive forces and penetration distance at the same time. In order to measure the resistive force and penetration distance, strain gauges and a rotary encoder were used, respectively. Two strain gauges were attached on the arm of LHD model. The rotary encoder was installed at the side of the body of the LHD. The signals from the strain gauges were transmitted to the personal computer through a strain meter and an $\mathrm{A} / \mathrm{D}$ converter. The $\mathrm{LHD}$ model was pushed manually.
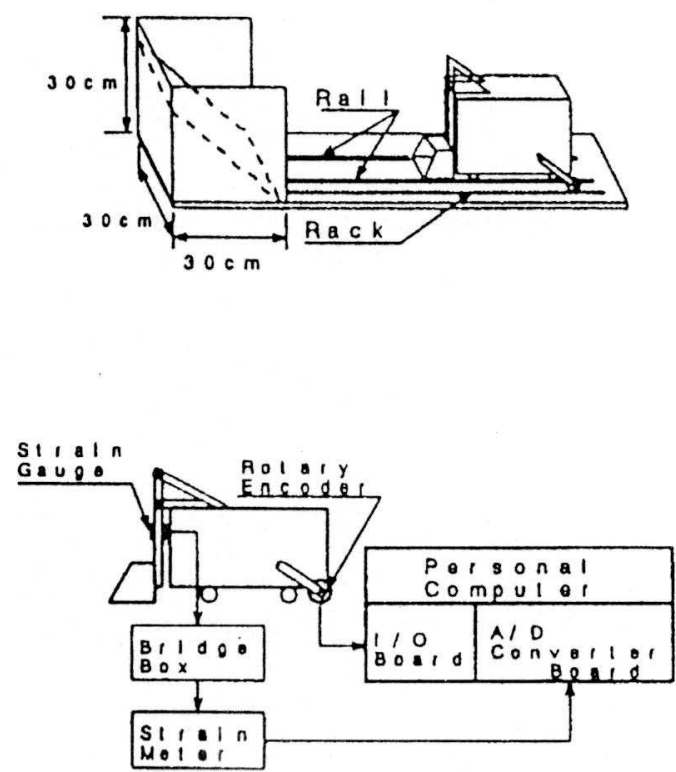

Fig.9 Schematic of experimental apparatus (Small model)

Particles used in this experiment were eight kinds of crushed rocks having different diameters, quartz

\begin{tabular}{|c|c|c|c|c|c|c|c|}
\hline No & Particle & $\rho$ & $\mathrm{ds}$ & $\varepsilon$ & $\phi_{\mathrm{k}}$ & $\not l$ & $\phi$ \\
\hline 1 & \multirow{8}{*}{$\mathrm{CR}$} & \multirow{4}{*}{2.74} & 0.9 & 0.53 & 32 & 0.514 & \multirow{8}{*}{34,21} \\
\hline 2 & & & 1.9 & 0.52 & 33 & 0.53 & \\
\hline 3 & & & 2.9 & 0.49 & 34 & 0.54 & \\
\hline 4 & & & 5.7 & 0.47 & 35 & 0.56 & \\
\hline 5 & & \multirow{4}{*}{2.50} & 5.8 & 0.52 & 36 & 0.506 & \\
\hline 6 & & & 8.0 & 0.51 & 39 & 0.521 & \\
\hline 7 & & & 12 & 0.50 & 40 & 0.545 & \\
\hline 8 & & & 20 & 0.49 & 43 & 0.587 & \\
\hline 9 & QS & 2.53 & 0.7 & 0.38 & 28 & 0.48 & 33,23 \\
\hline 10 & $\mathrm{PT}$ & 1.38 & 3.8 & 0.36 & 29 & 0.38 & 34,21 \\
\hline
\end{tabular}
sand and PVC pellet. The slopes of the rock piles were about 20 and 30 degrees. Properties of the particles and slope of the rock piles are listed in Table 1.

Table 1 Properties of particles used in the experiment and inclination angle of rock pile

CR:Crushed Rock, QS:Quartz Sand, PT:Pellet $\rho[\mathrm{kg} / \mathrm{m} 3]$ :density, ds[mm]:particle diameter, $\varepsilon[-]$ : porosity, $\phi_{k}[\mathrm{deg}$.]:friction angle among particles, $\mu[-]$ : friction coefficient between particles and bucket, $\phi[\mathrm{deg}$ ]]: inclination angle of rock pile

\section{Experimental Results}

Figure 10 shows an example of the mcasured results of the resistive forces. The horizontal axis shows the dimensionless distance, $\mathrm{X} / \mathrm{L}$. The vertical axis shows the resistive forces acting on the bucket, $F$. When $X / L$ is less than unity, $F$ increases gradually. 
On the other hand, when $\mathrm{X} / \mathrm{L}$ exceeds unity, F increases rapidly because the force due to the passive soil pressure increases rapidly. The solid line in Figure 10 shows the calculated results without $F_{3}$, because $F_{3}$ contains the unknown parameter, $\theta$. The calculated results are much smaller than the measured results. From this result, it can be demonstrated that the effect of the cutting force, $F_{3}$ on the total resistive forces is significant.

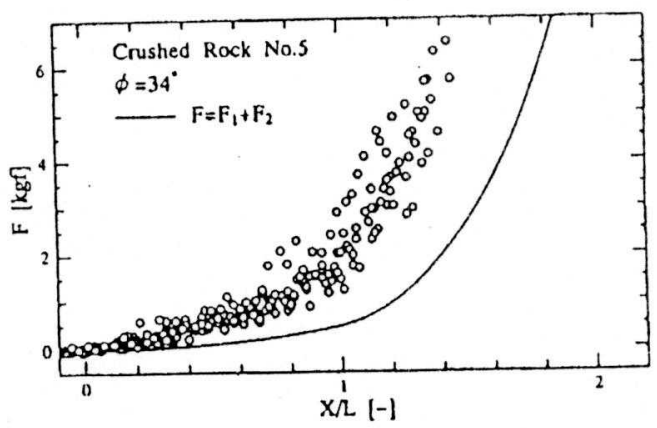

Fig.10 An example of measured resistive forces

If the calculation model of $\left(\mathrm{F}_{1}+\mathrm{F}_{2}\right)$ is correct, the difference between the measured results and the calculated $\left(\mathrm{F}_{1}+\mathrm{F}_{2}\right)$ should be equal to the cutting force, $F_{3}$. Therefore, the bucket as shown in Figure 11 was made to measure the cutting force, and the measurement of cutting forces was carried out.

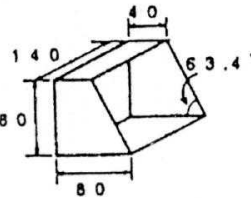

(a) Normal Bucko

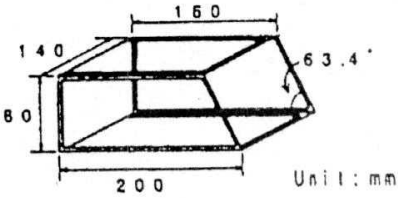

(6) Bucket lo Neasuta tho Culiting agsislire Eolo

Fig.11 Shape of the bucket to measure the cutting forces

Figure 12 shows the comparison between the measured cutting forces, $F_{3}$, and estimated cutting forces, $F-\left(F_{1}+F_{2}\right)$. Black and white symbols indicate the measured cutting forces, $F_{3}$, and estimated cutting forces, $\mathrm{F}-\left(\mathrm{F}_{1}+\mathrm{F}_{2}\right)$, respectively. Both symbols agree well with each other. So, it is confirmed that the models for $F_{1}$ and $F_{2}$ are reasonable.

In order to calculate the cutting force, $F_{3}$, it is neccssary to estimate the parameter $\theta$. As it was very difficult to estimate this parameter theoretically, the value of $\theta$ was obtained by the inverse calculation by using the measured results of the resistive forces.

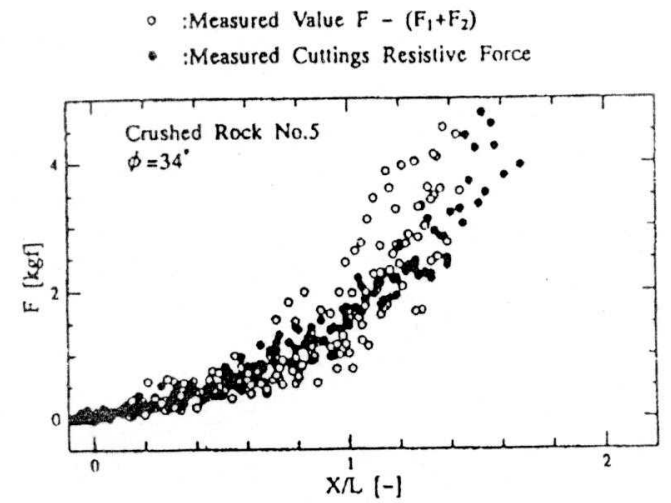

Fig.12 Comparison between F- $($ F1+F2) and measured cutting force

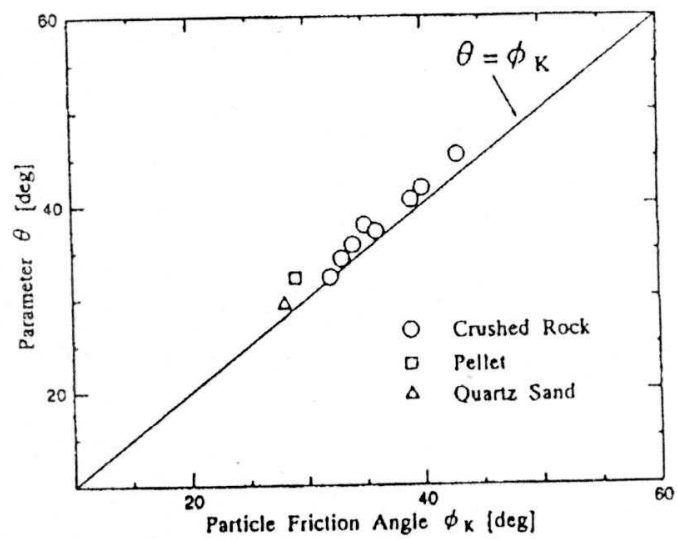

Fig.13 Relationship between friction angle among particles and parameter 0

Figure 13 shows the relationship between the friction angle among particles, $\phi_{k}$, and the parameter $\theta$. As shown in this figure, most of the values of $\theta$ fell close to the line of $\theta=\phi_{k}$, and they are a little larger than the friction angle among particles, $\phi_{\mathrm{k}}$.

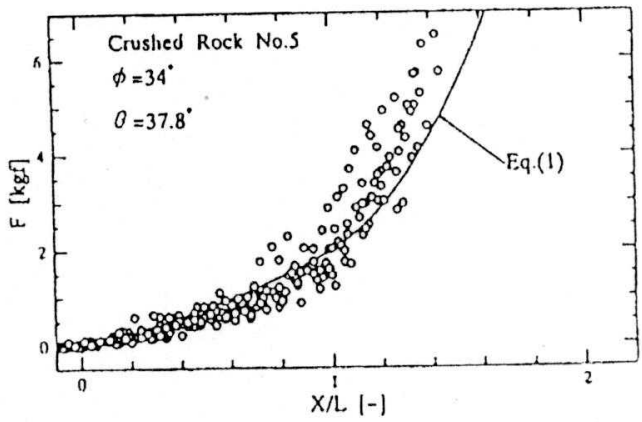

Fig. 14 Comparison between experimental results and calculated results

Figure 14 shows an example of the comparison 
between the measured resistive forces and calculated ones. The parameter, $\theta$ was estimated from Figure 13. As shown in Figure 14, the calculated results agreed well with the measured resistive forces. This result was the same regardless of the particles.

\section{Effect of Bucket Size}

In order to investigate the effect of the bucket size on the resistive forces, the experimental apparatus shown in Figure 15 was made. The capacity of the bucket in Figure 15 is about 50 times of the one in Figurc 9. The width of the bucket is $0.5 \mathrm{~m}$. The measurement procedure was the same as the one for the small experimental apparatus. The particles used in this experiment were $20 \mathrm{~mm}$ diameter crushed rock The slope of the rock pile was about 28 degrees.

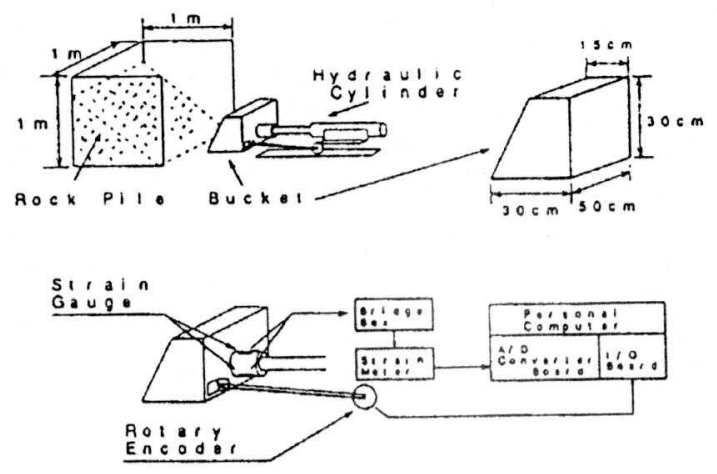

Fig.15 Schematic of experimental apparatus (Medium model)

Figure 16 shows an example of the measured resistive forces. The solid line in this figure shows the calculated results. The parameter, $\theta$ was estimated from Figure 13. As shown in this figure, the calculated results agreed well with the measured resistive forces. It was found from this figure that the model developed here is applicable to the large size bucket.

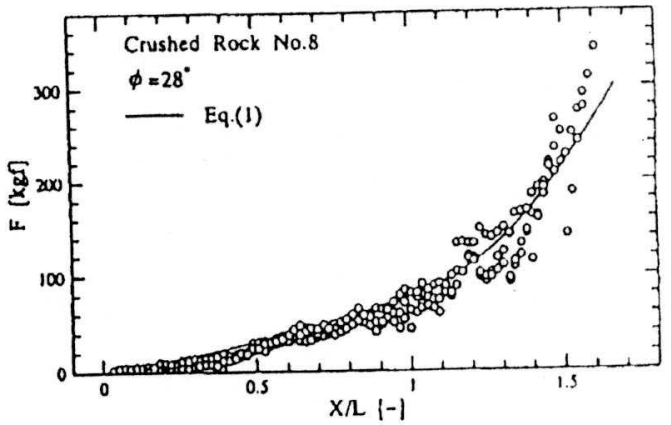

Fig.16 An example of measured resistive forces using medium model

\section{Conclusions}

In this study, the changes in shape of the rock pile due to penetration by the bucket were modeled. Based on this model, the resistive forces acting on the bucket were considered theoretically. Furthermore, the resistive forces were measured by using two kinds of LHD models. It was confirmed that the calculated results obtained using the model agree well with the experimental results.

\section{References}

1)M.St-Amant et al., : Proc. of 1st Int. Int Symposium on Mine Mechanization and Automation, pp.6/1-10, 1991.

2)P.H.King et al., : Proc. of lst Int. Int. Symposium on Mine Mechanization and Automation, pp.6/3144, 1991.

3)T.Gocho et al., : Proc. of 9th Int. Symposium on Automation and Robotics in Construction, pp.803812, 1992. 